\title{
Astroparticle Aspects of Supersymmetry
}

\author{
John Ellis ${ }^{\mathrm{a}}$

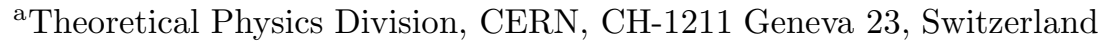

\begin{abstract}
After recalling the motivations for expecting supersymmetry to appear at energies $\lesssim 1 \mathrm{TeV}$, the reasons why the lightest supersymmetric particle is an ideal candidate for cold dark matter are reviewed from a historical perspective. Recent calculations of the relic density including coannihilations and rapid annihilations through direct-channel Higgs boson poles are presented. The experimental constraints from LEP and elsewhere on supersymmetric dark matter are reviewed, and the prospects for its indirect or direct detection are mentioned. The potential implications of a Higgs boson weighing about $115 \mathrm{GeV}$ and the recent measurement of the anomalous magnetic moment of the muon are summarized.
\end{abstract}

CERN-TH/2001-091 hep-ph/0103288

\section{Why Supersymmetry?}

As we have heard at this meeting, theorists have been attracted to supersymmetry for many different reasons: because it is there, because it is beautiful, to unify theories of matter and interactions, etc. However, these profound theoretical arguments have not been able to fix the energy scale where supersymmetry should appear, which could, a priori, be as large as the Planck scale $\sim 10^{19} \mathrm{GeV}$. On the other hand, an argument for fixing the masses of the supersymmetric partners of the Standard Model particles to be around $1 \mathrm{TeV}$ is provided by the chance offered by supersymmetry of making a large hierarchy of mass scales more natural [1].

By now, there are several circumstantial experimental hints in favour of supersymmetry at the $\mathrm{TeV}$ scale. One is the agreement of the gauge coupling strengths measured at LEP and elsewhere with supersymmetric grand unification, if the spartners of the Standard Model particles weigh $\sim 1 \mathrm{TeV}$ [2]. Another indication is provided by precision electroweak data, that favour a light Higgs boson [3], as predicted in the MSSM [4] ${ }^{1}$. Yet another motivation for new physics at the

\footnotetext{
${ }^{1}$ Later in this talk, the possibility that direct Higgs searches may be providing more than just an indication is also discussed [5].
}

TeV scale may have been provided recently by the anomalous magnetic moment of the muon [6], and supersymmetry is quite capable [7] of explaining the 2.6- $\sigma$ discrepancy between theory and the recent BNL E821 experiment [6].

In this talk, however, I should like to emphasize a third, astrophysical, motivation for TeVscale supersymmetry, namely the cold dark matter for which the lightest supersymmetric particle is an ideal candidate. Recall that conventional (baryonic) matter constitutes $\lesssim 5 \%$ of the critical density, according to analyses of Big-Bang nucleosynthesis [8] and the cosmic microwave background (CMB) radiation [9]. There are both astrophysical and particle-experimental reasons to believe that massive neutrinos could provide at most a similar percentage of the critical density [10]. However, analyses of the CMB and large-scale structure data suggest that the total amount of matter in the Universe corresponds to about $30 \%$ of the critical density, far more than could be provided by either baryons or neutrinos [9]. Theories of structure formation suggest that most of the matter density should be in the form of cold dark matter [11], such as massive weakly-interacting particles. The remaining $65 \%$ of the critical density, as required in inflationary cosmology, indicated by data on high-redshift supernovae [12] and supported by the CMB data [9], is presumably provided by some form of vacuum energy, either varying with time or a true cosmo- 
logical constant.

Turning to particle candidates for the cold dark matter, massive weakly-interacting particles that were originally in thermal equilibrium could have interesting cosmological densities if they had either (a) masses in the eV region, such as neutrinos, (b) masses of a few GeV, now excluded by LEP for particles with neutrino-like couplings to the $Z^{0}[13]$, or (c) masses in the range from $m_{Z} / 2$ up to about $1 \mathrm{TeV}$. It is the latter possibility that may be realized with the lightest supersymmetric particle (LSP).

In this talk, I first review how the realization grew that the LSP could be an excellent candidate for cold dark matter. Then I review the constraints on supersymmetric dark matter coming from accelerator experiments, principally those from LEP. Then I mention the principal strategies for searching for supersymmetric dark matter [14], including indirect searches for supersymmetric relic annihilations in the galactic halo or core, or inside the Sun or Earth, and direct searches for the scattering of dark matter particles on nuclei in the laboratory. Since one of the tightest constraints on the mass of the LSP is provided by the Higgs search at LEP, which has recently been the subject of much interest, I include a discussion of the status of Higgs searches at LEP. Also, I cannot resist adding some comments on the possible supersymmetric interpretation [7] of the recent BNL E821 measurement of the muon anomalous magnetic moment [6].

\section{The Lightest Supersymmetric Particle}

In many supersymmetric models, this is expected to be stable [15], and hence likely to be present today in the Universe as a cosmological relic from the Big Bang. It is stable because of a multiplicatively-conserved quantum number called $R$ parity [15], which takes the value +1 for all conventional particles and -1 for all sparticles. Its conservation is linked to those of baryon and lepton numbers:

$R=(-1)^{3 B+L+2 S}$

where $S$ is the spin. It is certainly possible to violate $R$ by violating $L$ either spontaneously or explicitly, but these options are limited by laboratory [16] and cosmological constraints [17], and we discard them for the rest of this talk.

The conservation of $R$ parity has the following three important consequences.

1) Sparticles are always produced in pairs, e.g., $p p \rightarrow \tilde{q} \tilde{q}+X$ or $e^{+} e^{-} \rightarrow \tilde{\mu}^{+} \tilde{\mu}^{-}$.

2) Heavier sparticles decay into lighter ones, e.g., $\tilde{q} \rightarrow q \tilde{q}$ or $\tilde{\mu} \rightarrow \mu \tilde{\gamma}$.

3) The lightest supersymmetric particle is stable, because it has no legal decay mode.

It is the latter property that makes the LSP an ideal particle candidate for dark matter.

Although Fayet [15] had appreciated previously the significance of $R$ parity, and he and Farrar [18] had discussed the phenomenology in laboratory experiments of the LSP, the first discussion of supersymmetric cold dark matter was by Goldberg [19], as far as I am aware. He discussed the case where the LSP is a pure photino $\tilde{\gamma}$, and linked its possible mass to those of the squarks. The next two papers known to me were [20] and [21]. We saw the Goldberg paper [19] while we were thinking how to search for supersymmetry at the CERN $\bar{p} p$ collider, and were impressed by his argument that cosmology imposed important constraints. However, we also realized that the pure photino limit he considered was insufficient, since this state must mix with the spartners of the neutral Higgs bosons and the $Z^{0}$, in any realistic model. We added some consideration of this mixing in our collider paper [20], and resolved to study in more detail the full case of mixing between the $\tilde{\gamma}, \tilde{H}$ and $\tilde{Z}^{0}$ in a generic neutralino $\chi$. The resulting paper [22] came out a few months later.

In addition to calculations of the neutralino relic density, we also presented in [22] some general arguments on the likely nature of the LSP, in particular why it should interact only weakly. If the supersymmetric relic had either electric charge or strong interactions, it would have condensed into ordinary matter, and shown up as an anomalous heavy isotope. These have not been seen, and experiments impose [23]

$\frac{n(\text { relic })}{n(p)} \lesssim 10^{-15}$ to $10^{-30}$ 
for $1 \mathrm{GeV} \lesssim m_{\text {relic }} \lesssim 10 \mathrm{TeV}$, far below the expected supersymmetric relic abundance. We conclude that the supersymmetric relic is surely electrically neutral and weakly-interacting, e.g., it cannot be a gluino [22]. The latter possibility is resuscitated occasionally, but we are convinced that it is excluded by this anomalous-isotope argument.

Looking through the sparticle data book, the possible cold dark matter scandidates are the sneutrinos $\tilde{\nu}$ of spin 0 , the lightest neutralino $\chi$ of spin $1 / 2$, and the gravitino $\tilde{G}$ of spin $3 / 2$. Sneutrinos are excluded by a combination of LEP and direct searches. The gravitino is the LSP in gauge-mediated models, in particular, but would only provide hot (or, at best, warm) dark matter, rather than cold, so we do not discuss it further here.

The lightest neutralino $\chi$ is therefore the favoured dark matter candidate in many supersymmetric models. At the tree level, it is characterized by 3 parameters: the unmixed gaugino mass, $m_{1 / 2}$, assumed to be universal, the Higgs mixing parameter, $\mu$, and the ratio of Higgs v.e.v.'s, $\tan \beta[22]$. The neutralino composition simplifies in the limit $m_{1 / 2} \rightarrow 0$, where it becomes an almost pure photino $\tilde{\gamma}$ [19], and in the limit $\mu \rightarrow 0$, where it becomes an almost pure Higgsino $\tilde{H}$ [20]. However, both of these limits are excluded by LEP, which enforces $m_{\chi} \gtrsim 100$ $\mathrm{GeV}[24,5,25]$ and non-trivial mixing, as we discuss in more detail below.

One of the most exciting features of neutralino dark matter is that there are generic domains of parameter space where an 'interesting' cosmological relic density: $0.1 \lesssim \Omega_{\chi} h^{2} \lesssim 0.3$ is possible for some suitable choice of the other supersymmetric model parameters $[22,24]$. This point will be discussed in more detail below, after further discussion of the experimental and cosmological constraints on supersymmetric dark matter.

\section{Experimental and Cosmological Con- straints}

The most direct limits on supersymmetric dark matter come from direct searches for other supersymmetric particles. For example, LEP has established that charginos $\chi^{ \pm}$weigh $\geq$ 103.5 GeV [26], and has also established important limits by searching for the associated production of neutralinos: $e^{+} e^{-} \rightarrow \chi+\chi^{\prime}$. LEP has also established a lower limit of $100 \mathrm{GeV}$ on the selectron mass, as seen in Fig. 1 [27]. However, one of the most stringent sparticle limits comes indirectly from the Higgs search [28], since [24] the Higgs mass is sensitive to sparticle masses via radiative corrections [4]:

$\delta m_{h}^{2} \propto \frac{m_{t}^{4}}{m_{W}^{2}} \ln \left(\frac{m_{\tilde{t}}^{2}}{m_{t}^{2}}\right)$

Because of the sensitivity to $m_{\tilde{t}}$ in (2), experimental constraints on squarks, in particular the lighter stop: $m_{\tilde{t}_{1}} \geq 95 \mathrm{GeV}$, as seen in Fig. 2 [29], also impact the neutralino limits.

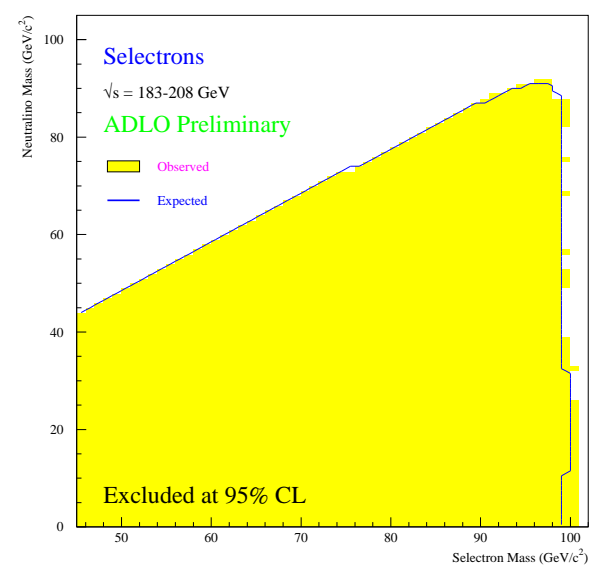

Figure 1. LEP constraint on the selectron, in the $m_{\tilde{e}}, m_{\chi}$ plane [27].

Relating the Higgs and the direct experimental limits on different types of sparticles requires making some hypotheses on the space of supersymmetric parameters. The minimal supersymmetric extension of the Standard Model (MSSM) 
4

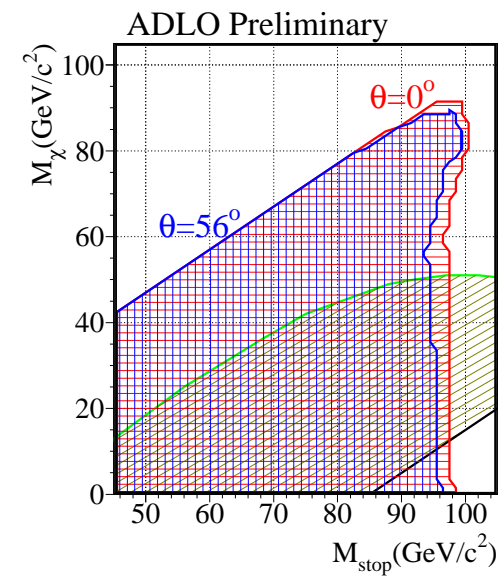

Figure 2. LEP constraints on the lighter stop $\tilde{t}_{1}$, in the $m_{\tilde{t}_{1}}, m_{\chi}$ plane, for various mixing scenarios [29].

is generally assumed, and sample MSSM Higgs limits are shown in Fig. 3. These are calculated in a maximal mixing scenario, in which the $Z Z h$ coupling may be suppressed at large $\tan \beta$, relaxing the lower limit on $m_{h}$ from its Standard Model value. In this talk I further assume the constrained MSSM (CMSSM), in which the soft supersymmetry-breaking scalar and fermionic mass parameters $m_{1 / 2}$ and $m_{0}$ are universal at some input GUT scale, as are the soft trilinear supersymmetry-breaking parameters $A_{0}$. In this case, the $Z Z h$ coupling is not suppressed at large $\tan \beta$, and the LEP lower limit on the lightest CMSSM Higgs boson is essentially identical to that in the Standard Model. Representative examples of the sensitivity of neutralino limits to Higgs and other limits are shown in Figs. 4 and 5 [25], for $\mu>0$ and $\mu<0$, respectively.

We have included in Figs. 4 and 5 [25] the constraint imposed by the measurement of $b \rightarrow$ $s \gamma$ [30], when compared with the latest NLO QCD calculations valid for large $\tan \beta$ [31], which is shown as a mdedium-shaded region. These plots also include the requirement that the lighter stau, $\tilde{\tau}_{1}$, not be the LSP [24], which is disallowed because the LSP cannot be charged. Fig. 4(a) also shows the impact of LEP chargino searches. If one is nervous about the fate of our vacuum, one may also require that the effective potential have no charge- and colour-breaking (CCB) minimum [32]. However, this requirement may be regarded as optional: it is possible that our present electroweak vacuum may be unstable but longlived. Hence the CCB constraint is not shown in Figs. 4 and 5.

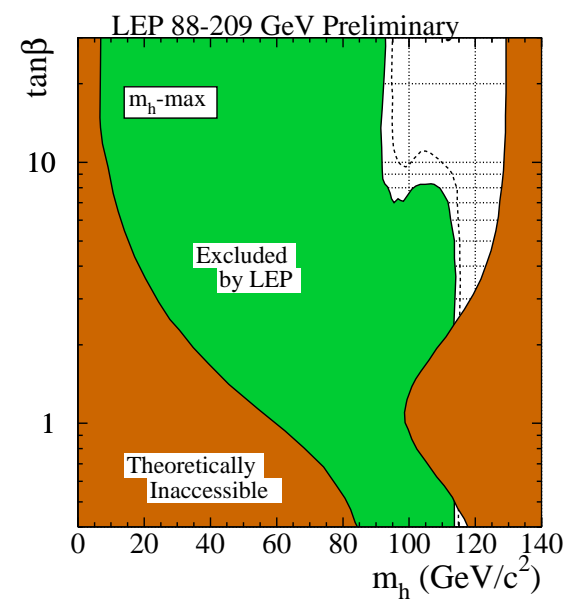

Figure 3. LEP constraints on MSSM Higgs bosons, in the $m_{h}, \tan \beta$ plane, assuming a maximal mixing scenario and neglecting $C P$ violation [28].

The light-shaded cosmological region in Figs. 4 and 5 has a 'tail' extending up to $m_{1 / 2} \sim 1400$ $\mathrm{GeV}$ for $\tan \beta \lesssim 20$, as seen in panels (a) of these figures, which is due to efficient $\chi-\tilde{\ell}$ coannihilations $[33,34]$. These increase the upper limit on the LSP mass to

$m_{\chi} \sim 600 \mathrm{GeV}$.

LHC appears able to find at least some supersymmetric particles over all the comsologicallyallowed range for $\tan \beta \lesssim 20$ [35]. However, for larger $\tan \beta$, the coannihilation region extends to larger $m_{1 / 2}$, as seen in panels (b) of Figs. 4 and 

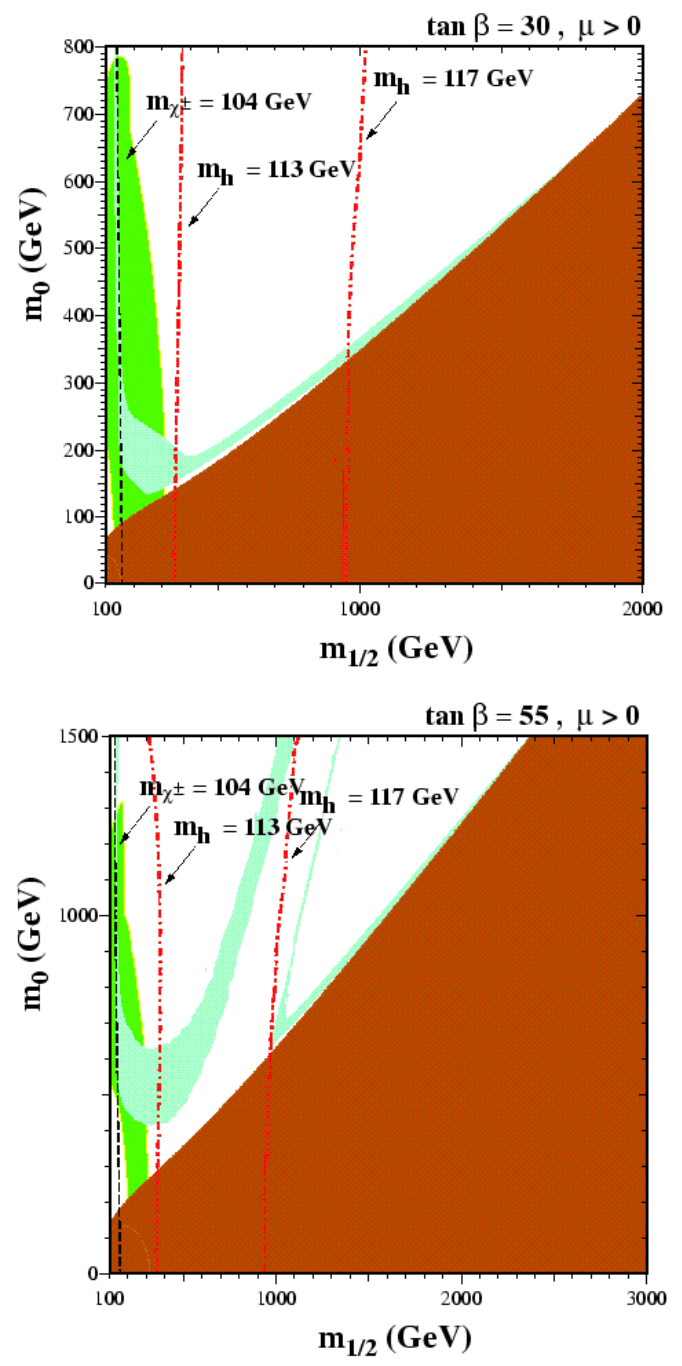

Figure 4. Domains of the $m_{0}, m_{1 / 2}$ plane in the CMSSM for $\mu>0$, and $\tan \beta=$ (a) 30, (b) 55 , showing the impacts of various experimental and cosmological constraints [25]. Vertical broken lines indicate Higgs mass contours, assuming universality of the soft supersymmetrybreaking scalar mass $m_{0}$ and gaugino mass $m_{1 / 2}$. Also shown in panel (a) is the impact of chargino searches at LEP. The dark shaded region is excluded because the lightest supersymmetric particle is the charged $\tilde{\tau}_{1}$, and the medium shaded region is excluded by the measured $b \rightarrow$ s $\gamma$ rate. The light shaded region is that preferred by cosmology, namely $0.1<\Omega h^{2}<0.3$.
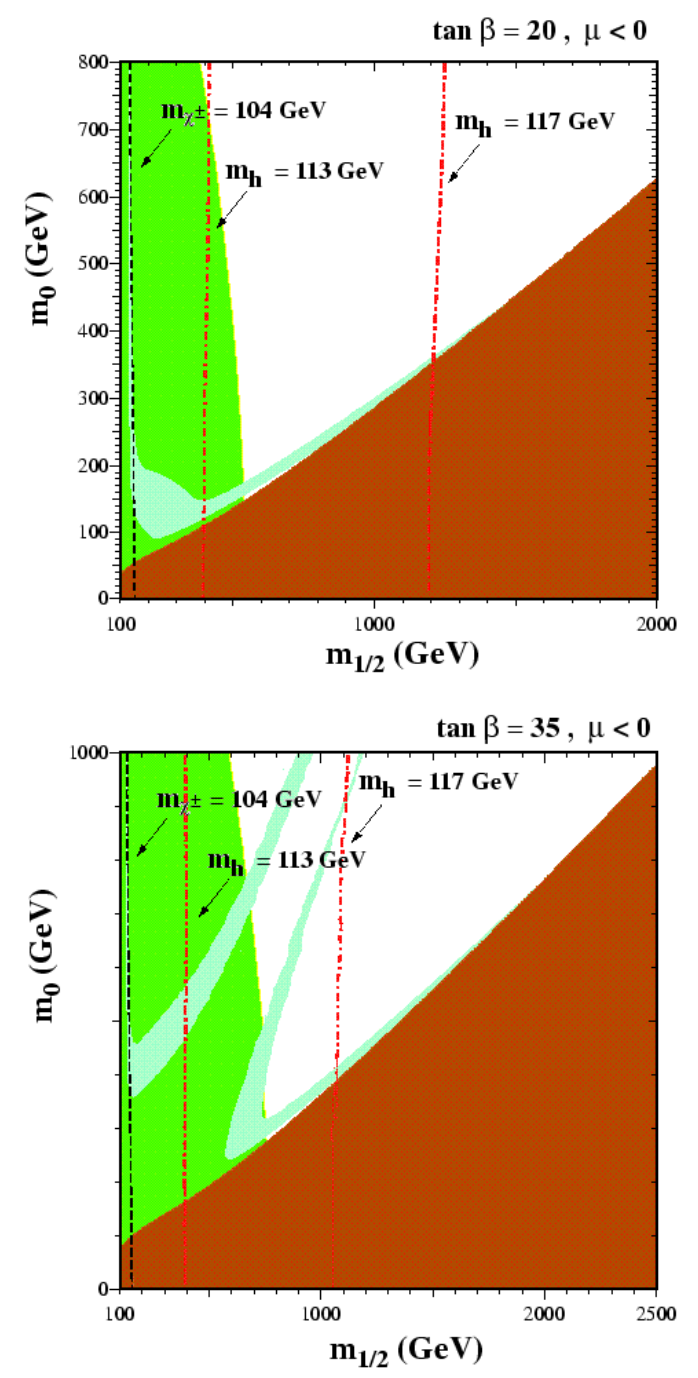

Figure 5. Domains of the $m_{0}, m_{1 / 2}$ plane in the CMSSM for $\mu<0$ and $\tan \beta=$ (a) 20, (b) 35, showing the impacts of various experimental and cosmological constraints [25]. Notations are the same as in Fig. 4. 
$5^{2}$. Hence, the 'guarantee' of supersymmetric discovery at the LHC does not extend to such larger values of $\tan \beta$. Another feature visible for larger $\tan \beta$ is a double 'funnel' region of allowed LSP density extending out to large $m_{0}$ and $m_{1 / 2}$, which occurs because of rapid $\chi \chi \rightarrow A, H$ annihilation. This also extends beyond the region of 'guaranteed' discovery at the $\mathrm{LHC}^{3}$.

The constraints from LEP and $b \rightarrow s \gamma$ impose a lower limit on $m_{\chi}$ within this cosmological regionw. Its strength depends on $\tan \beta$ and theoretical assumptions, but we find in general that [25]

$m_{\chi} \gtrsim 100 \mathrm{GeV}$ and $\tan \beta \gtrsim 3$

with the precise numbers depending on the sign of $\mu$ and different assumptions, e.g., on the values of $m_{t}, m_{b}$ and $A_{0}$. If the pole mass $m_{t}=175 \mathrm{GeV}$, the running mass $m_{b}\left(m_{b}\right)_{S M}^{\bar{M} S}=4.25 \mathrm{GeV}$ and $A_{0}=0$, we find $m_{\chi} \gtrsim 140(180) \mathrm{GeV}$ and $\tan \beta \gtrsim$ $5(7)$ for $\mu>(<) 0$, as seen in Fig. $6{ }^{4}$.

Within the region accessible to the LHC, one may ask whether it will find supersymmetry before the searches for astrophysical dark matter ${ }^{5}$.

\section{Searches for Dark Matter}

One strategy is to look for the annihilations of relic particles in the galactic halo [37], which may yield observable fluxes of (stable) particles such as $\bar{p}, \gamma$ and $e^{+}$. Measurements of the low-energy $\bar{p}$ flux already rule out some supersymmetric models [38]. On the other hand, $\gamma$ searches do not yet rule out any models, unless the relic density in our galactic halo is strongly clumped [39]. There have been some reports of an excess of cosmic-ray positrons, but some of these have now been contradicted, and they cannot be taken as evidence for supersymmetric dark matter.

\footnotetext{
${ }^{2}$ In these panels, we have chosen the largest values of $\tan \beta$ for which we consistent electroweak vacua with our default choices of auxiliary parameters $m_{t}=175 \mathrm{GeV}$, $m_{b}\left(m_{b}\right)_{S M}^{\bar{M} S}=4.25 \mathrm{GeV}$ and $A=0$.

${ }^{3}$ At least within the CMSSM, sparticle searches at Run II of the FNAL Tevatron collider will not be able to probe much of the parameter space not already excluded [36].

${ }^{4}$ Note, also, that the bound (5) could be relaxed if the soft supersymmetry-breaking scalar masses are not universal.

${ }^{5}$ As we see later, the E821 measurement [6] offers some hope of restoring the 'guarantee' that the LHC will find supersymmetry.
}

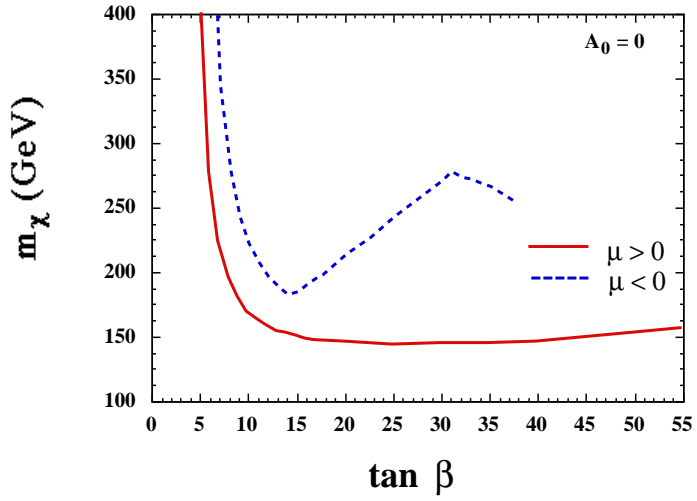

Figure 6. Lower limits on the mass of the lightest supersymmetric particle in the CMSSM, assuming $m_{t}=175 \mathrm{GeV}, m_{b}\left(m_{b}\right)_{S M}^{\bar{M} S}=4.25 \mathrm{GeV}$ and $A_{0}=0$.

Another search strategy is to look for annihilations of relic particles inside the Sun or Earth, following their capture after losing energy via elastic scattering [40]. The observable products of these annihilations are high-energy neutrinos, which may either be seen directly in underground detectors, or indirectly via $\nu$ collisions in rock that produce detectable muons. Searches for neutrinoinduced muons from the Sun and Earth already exclude a significant number of supersymmetric models, and there are prospects for improving the current search sensitivities with a $1 \mathrm{~km}^{2}$ (or 1 $\mathrm{km}^{3}$ ) detector [41].

The third favoured strategy is to search directly for elastic dark matter scattering on nuclei in the laboratory [42]. Each scattering event would deposit typically an energy $E \sim m_{\chi} b^{2} / 2 \sim$ tens of keV. There are two important types of interaction: spin-dependent, which is sensitive to the different quark contributions to the nucleon spin, and spin-independent, which is sensitive to the quark contributions to the nucleon mass. The former is likely to be more important for light nuclei, the latter for heavy nuclei.

One experiment reports annual modulation of the rate of energy deposition in their detector which may be interpreted as a possible signal 

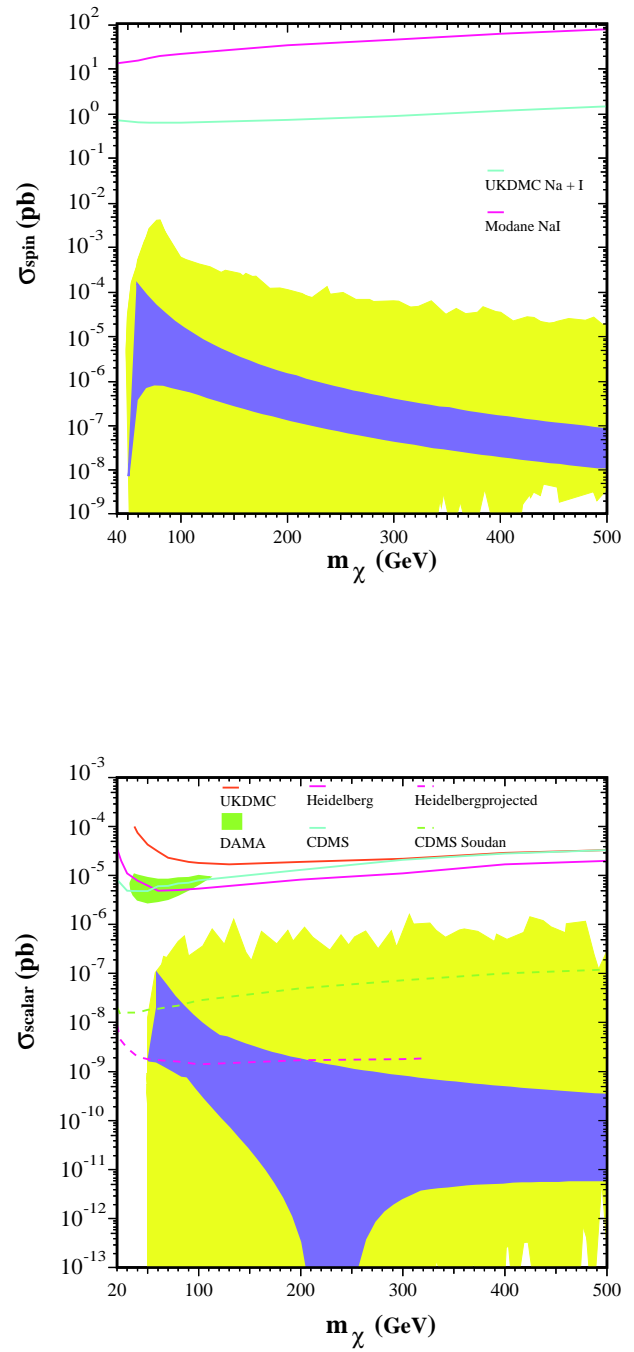

Figure 7. Compilations of ranges of the elastic cross sections found in a sampling of supersymmetric models for $\tan \beta \leq 10$, compared with the sensitivity of the DAMA [43] and other experiments, for spin-independent scattering. The dark (light) shaded regions correspond to CMSSM (MSSM) models with (without) universal soft scalar supersymmetry-breaking masses [46]. for dark matter scattering [43]. We recently reevaluated the rate of elastic scattering in a constrained supersymmetric model, in which all the soft scalar supersymmetry-breaking masses are assumed equal at the input unification scale [44]. As seen in Fig. 7, we found an elastic scattering cross-section below the region of experimental interest, at least for $\tan \beta \leq 10$. Subsequently, a second experiment published an upper limit that excluded most of the interesting region [45]. We have looked again at the elastic scattering rate, this time relaxing the assumption of universal scalar masses [46]. Although the rates could be somewhat higher than in the universal case, they still fell short of the region of experimental interest, at least for $\tan \beta \leq 10[47]^{6}$.

\section{What if ...?}

On September 5th, the ALEPH experiment at LEP reported an apparent excess of Higgs boson candidates in the reaction $e^{+} e^{-} \rightarrow(H \rightarrow$ $\bar{b} b)+(Z \rightarrow \bar{q} q)$. Two of the ALEPH events were rated as very good candidates that were quite unlikely to be due to $e^{+} e^{-} \rightarrow Z+Z$, the most dangerous background reaction. However, this background cannot be completely removed. During the period up to Nov. 2nd, while LEP continued running, the LEP Higgs 'signal' grew approximately as would be expected if there was a Higgs boson weighing about $115 \mathrm{GeV}$, with indications in other channels and in other experiments [49]. However, a preliminary estimate of the overall significance of the reported signal only amounted to at most 2.9 standard deviations [50], not enough for LEP to claim discovery. Nevertheless, one may speculate on the interpretation of any signal of a Higgs boson weighing around 115 $\mathrm{GeV}[5]$.

The first implication is that the Standard Model must break down at some scale $\lesssim 10^{6} \mathrm{GeV}$, with the appearance of some new bosonic particles. This is because the Standard Model Higgs potential becomes unstable at a scale $10^{6} \mathrm{GeV}$,

${ }^{6}$ We have recently extended our analysis to larger $\tan \beta$ in the CMSSM [48], incorporating the latest LEP experimental constraints, and find cross sections in a similar range as before. 
because the radiative corrections due to such a light Higgs boson are overwhelmed by those due to the relatively heavy top quark. What sort of new physics may appear? Technicolour [51] and other models with strongly-interacting Higgs bosons are excluded yet again [52], because they generically predict a heavier Higgs boson: 300 $\mathrm{GeV} \lesssim m_{H} \lesssim 1 \mathrm{TeV}$. On the other hand, such a light Higgs boson is completely consistent with supersymmetry, which predicts that the lightest Higgs boson weighs $\lesssim 130 \mathrm{GeV}$ [4].

Moreover, it can be argued that the new bosonic physics must quack very much like supersymmetry [53]. It has to be quite finely tuned, if the effective potential is not to blow up or become unbounded below. Such fine tuning is natural in supersymmetry, but would be lost, e.g., if there were no supersymmetric Higgsino partners of the Higgs boson. The discovery of a Higgs boson weighing $115 \mathrm{GeV}$ would therefore strengthen significantly the circumstantial phenomenological case for supersymmetry.

In fact, as seen in Fig. 8, a measurement of a Higgs mass around $115 \mathrm{GeV}$ could be used to estimate the value of $m_{1 / 2}$ required in the radiative correction (3). This is compatible with the range given earlier (5): if the present 'signal' does not eventually evaporate, the LSP mass may be quite close to the lower limit shown in Fig. 6 as a function of $\tan \beta$. The principal uncertainty in the estimate of $m_{\chi}$ is that due to uncertainties in calculating $m_{h}$, in particular that due to the range $m_{t}=175 \pm 5 \mathrm{GeV}$, since $\delta m_{h} / \delta m_{t}=\mathcal{O}(1)$.

As seen in Fig. 9, this value of $m_{h}$ is compatible with $m_{1 / 2} \lesssim 1400 \mathrm{GeV}$, as favoured by supersymmetric dark matter. It is possible to strengthen the upper limit on $m_{1 / 2}$ if $m_{h}=115 \mathrm{GeV}$, $m_{t}=175$ or $180 \mathrm{GeV}$ and $\tan \beta$ is sufficiently large, but not if $m_{t}=170 \mathrm{GeV}$. Of course, these upper limits would evaporate with the Higgs signal.

Finally, I cannot resist adding as an anachronistic addendum a short discussion of the possible evidence for physics beyond the Standard Model provided by the recent BNL E821 measurement of the anomalous magnetic moment of the muon. Prima facie, the discrepancy in this measurement is evidence for new physics at the $\mathrm{TeV}$ scale, and
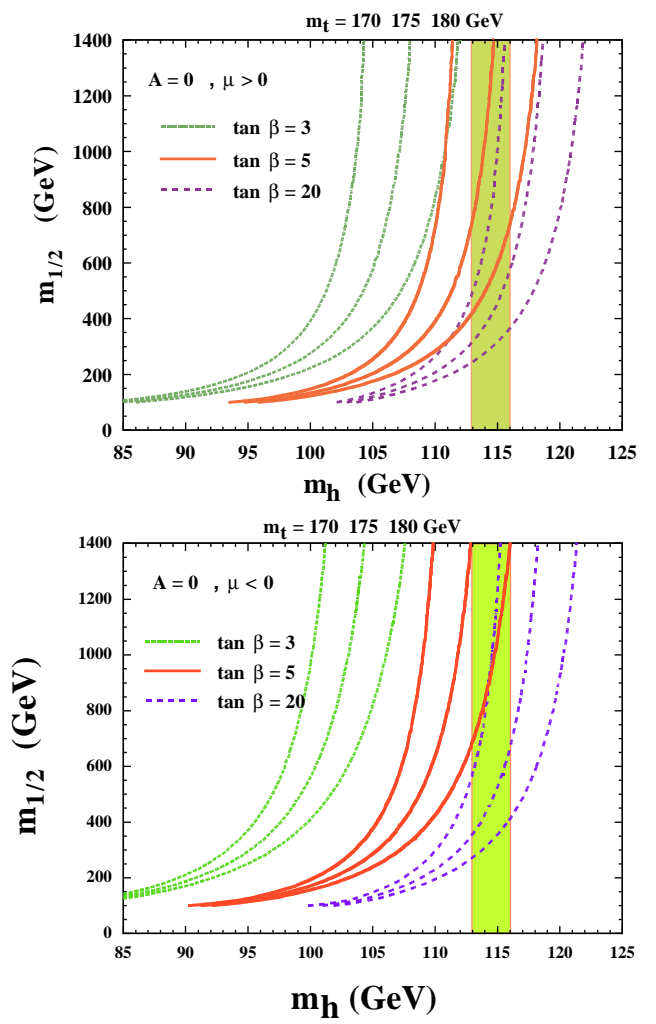

Figure 8. The sensitivity of $m_{h}$ to $m_{1 / 2}$ in the CMSSM for (a) $\mu>0$ and (b) $\mu<0$. The value $A_{0}=0$ is assumed for definiteness. The dotted (green), solid (red) and dashed (blue) lines are for $\tan \beta=3,5$ and 20, each for $m_{t}=170,175$ and $180 \mathrm{GeV}$ (from left to right). The lines are relatively unchanged as one varies $\tan \beta \gtrsim 10$, where they are also insensitive to the sign of $\mu$. The shaded vertical strip corresponds to $113 \mathrm{GeV} \leq$ $m_{h} \leq 116 \mathrm{GeV}$. 

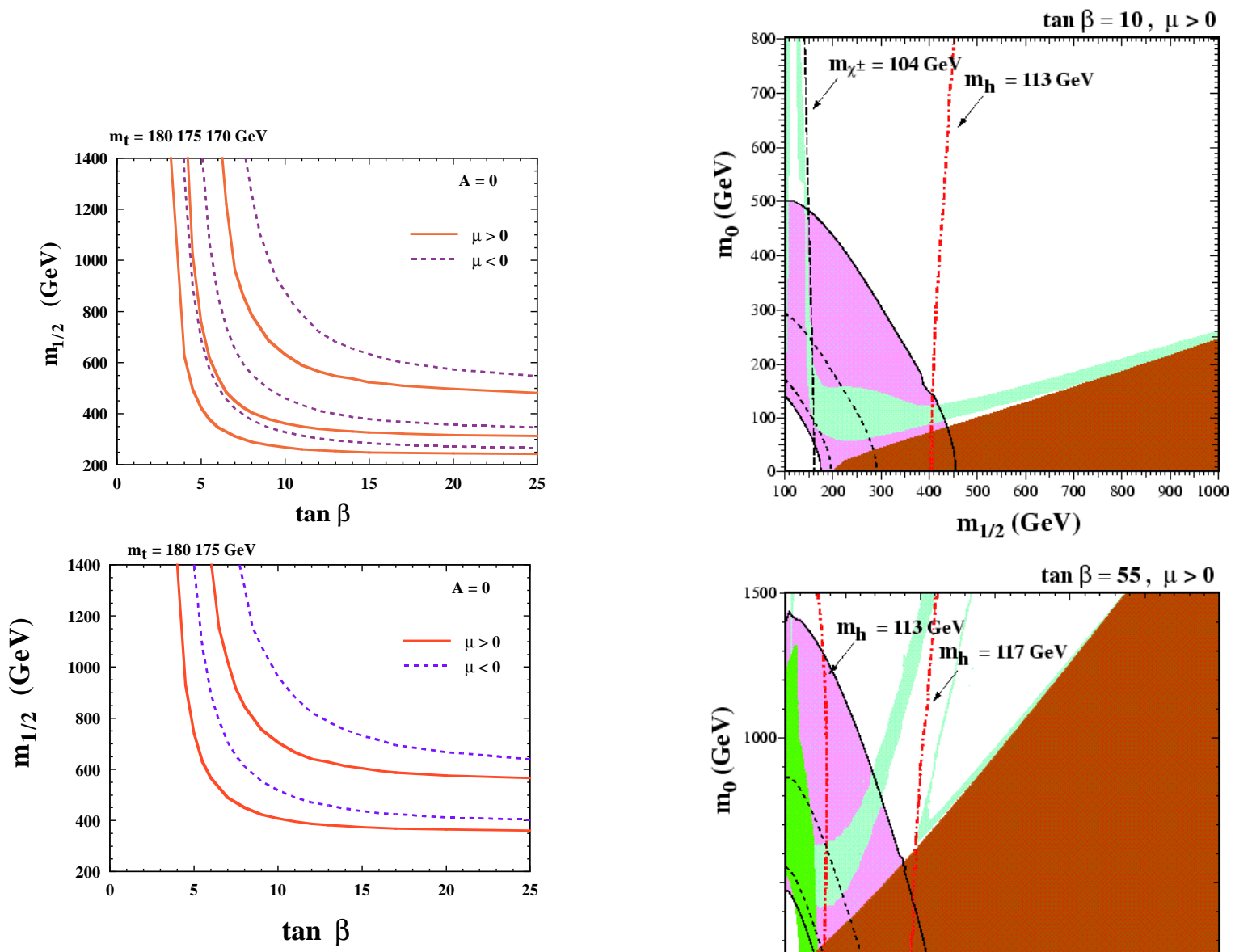

Figure 9. (a) The lower limit on $m_{1 / 2}$ required in the CMSSM to obtain $m_{h} \geq 113 \mathrm{GeV}$ for $\mu>0$ (solid, red lines) and $\mu<0$ (dashed, blue lines), and $m_{t}=170,175$ and $180 \mathrm{GeV}$, and (b) the upper limit on $m_{1 / 2}$ required to obtain $m_{h} \leq 116 \mathrm{GeV}$ for both signs of $\mu$ and $m_{t}=175$ and $180 \mathrm{GeV}$ : if $m_{t}=170 \mathrm{GeV}, m_{1 / 2}$ may be as large as the cosmological upper limit $\sim 1400 \mathrm{GeV}$. The corresponding values of the lightest neutralino mass $m_{\chi} \simeq 0.4 \times m_{1 / 2}$.

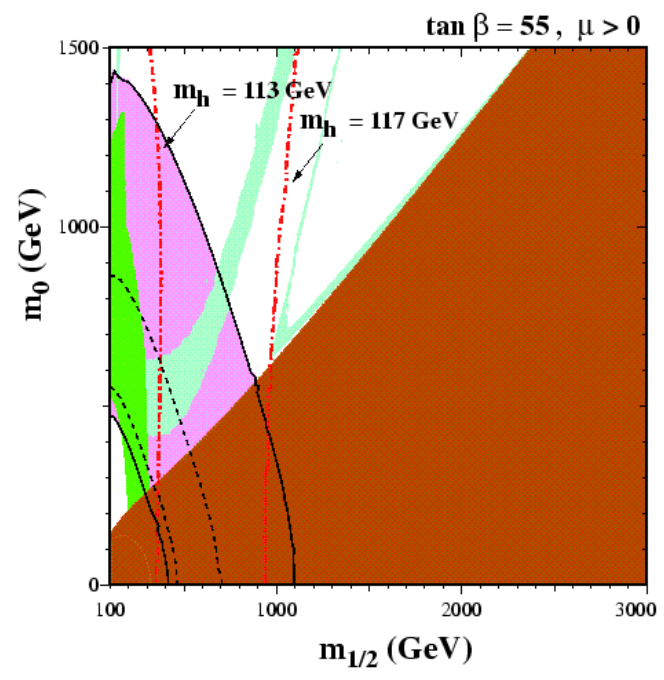

Figure 10. Superpositions on the CMSSM $\left(m_{1 / 2}, m_{0}\right)$ planes for $\mu>0$ and $\tan \beta=10,55$, see Fig. 4, of the constraint imposed by the E821 measurement of $a_{\mu}$. The regions allowed at the 2- $\sigma$ level are shaded (pink) and bounded by solid black lines, with dashed lines indicating the 1- $\sigma$ ranges. 
supersymmetry could well explain the E821 result, if $\mu>0$. Fig. 10 compares the regions of the CMSSM parameter space allowed by cosmology, the LEP sparticle exclusions, the Higgs 'signal' and $b \rightarrow s \gamma$ decay with that suggested by the E821 measurement. There is good consistency for $\tan \beta \gtrsim 10$ and moderate values of $m_{1 / 2}$ and $m_{0}$, well within the reach of the LHC. The BNL E821 measurement does not change the lower limit on $m_{1 / 2}$ and hence $m_{\chi}$ reported earlier, but it would, if confirmed, strengthen the upper limit (4) to about $400 \mathrm{GeV}$.

\section{How many Swallows?}

We have seen in this talk many indirect indications that the summer of supersymmetry may be on its way: the hierarchy problem, the unification of gauge couplings, a (possible) light Higgs boson, cosmological dark matter, and most recently the anomalous magnetic moment of the muon. These all encourage us theorists who have been hibernating through 30 years of supersymmetric winter. However, confirmation of our speculations can only come from the direct detection of supersymmetric particles, either in an accelerator experiment or as part of the astrophysical dark matter. If the non-accelerator experiments do not find it first, I am optimistic that the LHC will find supersymmetry, assuming that our phenomenological motivations for expecting it at the $\mathrm{TeV}$ scale have any validity. If all goes well, the LHC should be able to confirm or refute our speculations by the end of the decade. Compared with the 30 years we have already waited, perhaps that should not seem so long to wait.

\section{Acknowledgements}

It is a pleasure to thank my many collaborators on the subjects discussed here, most particularly Keith Olive.

\section{REFERENCES}

1. L. Maiani, Proceedings of the 1979 Gif-surYvette Summer School On Particle Physics, 1; G. 't Hooft, in Recent Developments In Gauge Theories, Proceedings of the Nato Advanced Study Institute, Cargese, 1979, eds.
G. 't Hooft et al., (Plenum Press, NY, 1980);

E. Witten, Phys. Lett. B105 (1981) 267.

2. J. Ellis, S. Kelley and D. V. Nanopoulos, Phys. Lett. B249 (1990) 441 and Phys. Lett. B260 (1991) 131; U. Amaldi, W. de Boer and H. Furstenau, Phys. Lett. B260 (1991) 447; C. Giunti, C. W. Kim and U. W. Lee, Mod. Phys. Lett. A6 (1991) 1745; P. Langacker and M. Luo, Phys. Rev. D44 (1991) 817.

3. LEP Electroweak Working Group, http://lepewwg.web.cern.ch/LEPEWWG/ stanmod/.

4. J. Ellis, G. Ridolfi and F. Zwirner, Phys. Lett. B257 (1991) 83; M.S. Berger, Phys. Rev. D41 (1990) 225; Y. Okada, M. Yamaguchi and T. Yanagida, Prog. Theor. Phys. 85 (1991) 1; Phys. Lett. B262 (1991) 54; H.E. Haber and R. Hempfling, Phys. Rev. Lett. 66 (1991) 1815.

5. J. Ellis, G. Ganis, D. V. Nanopoulos and K. A. Olive, Phys. Lett. B502 (2001) 171.

6. H. N. Brown et al., BNL E821 Collaboration, Phys. Rev. Lett. 86 (2001) 2227.

7. J. Ellis, D. V. Nanopoulos and K. A. Olive, hep-ph/0102331. For other supersymmetric analyses of the E821 result, see: L. Everett, G. L. Kane, S. Rigolin and L. Wang, hepph/0102145; J. L. Feng and K. T. Matchev, hep-ph/0102146; E. A. Baltz and P. Gondolo, hep-ph/0102147; U. Chattopadhyay and P. Nath, hep-ph/0102157; S. Komine, T. Moroi and M. Yamaguchi, hep-ph/0102204; R. Arnowitt, B. Dutta, B. Hu and Y. Santoso, hep-ph/0102344; T. Kobayashi and H. Terao, hep-ph/0103028; K. Choi, K. Hwang, S. K. Kang and K. Y. Lee, hep-ph/0103048; S. P. Martin and J. D. Wells, hep-ph/0103067; S. Komine, T. Moroi and M. Yamaguchi, hepph/0103182; K. Cheung, C. H. Chou and O. C. W. Kong, hep-ph/0103183; S. Baek, P. Ko and H. S. Lee, hep-ph/0103218.

8. K. A. Olive, G. Steigman and T. P. Walker, Phys. Rept. 333-334 (2000) 389.

9. A. E. Lange et al., astro-ph/0005004.

10. J. Ellis, summary talk at the XIXth International Conference on Neutrino Physics and Astrophysics, Sudbury, Canada, June 2000: hep-ph/0008334 and references therein. 
11. For a review, see: E. Gawiser and J. Silk, Science280 (1998) 1405.

12. See, for example, N. Bahcall, J. P. Ostriker, S. Perlmutter and P. J. Steinhardt, Science 284 (1999) 1481.

13. J. Ellis, D. V. Nanopoulos, L. Roszkowski and D. N. Schramm, Phys. Lett. B245 (1990) 251.

14. For a review, see: L. Bergstrom, Rept. Prog. Phys. 63 (2000) 793.

15. See, in particular, P. Fayet, Phys. Lett. 69B (1977) 489. For a review of his early work, see: P. Fayet, in Proceedings of the Europhysics Study Conf. on Unification of Fundamental Interactions, Erice, Italy, Mar 17-24, 1980.

16. See, for example: H. Dreiner, hep$\mathrm{ph} / 9707435$.

17. B. A. Campbell, S. Davidson, J. Ellis and K. A. Olive, Phys. Lett. B 256 (1991) 457; W. Fischler, G. F. Giudice, R. G. Leigh and S. Paban, Phys. Lett. B 258 (1991) 45.

18. G. R. Farrar and P. Fayet, Phys. Lett. 76B (1978) 575 and 79B (1978) 442.

19. H. Goldberg, Phys. Rev. Lett. 50 (1983) 1419.

20. J. Ellis, J. S. Hagelin, D. V. Nanopoulos and M. Srednicki, Phys. Lett. B127 (1983) 233.

21. L. M. Krauss, Nucl. Phys. B227 (1983) 556.

22. J. Ellis, J.S. Hagelin, D.V. Nanopoulos, K.A. Olive and M. Srednicki, Nucl. Phys. B238 (1984) 453.

23. See, for example, J. L. Basdevant, R. Mochkovitch, J. Rich, M. Spiro and A. Vidal-Madjar, Phys. Lett. B234 (1990) 395.

24. J. Ellis, T. Falk, G. Ganis and K. A. Olive, hep-ph/0004169.

25. J. Ellis, T. Falk, G. Ganis and K. A. Olive, Phys. Rev. D62 (2000) 075010.

26. LEP2 SUSY Working Group, http://lepsusy.web.cern.ch/lepsusy/ www/inos_moriond01/charginos_pub.html.

27. LEP2 SUSY Working Group, http://alephwww.cern.ch/ ganis/SUSYWG/ SLEP/sleptons_2k01.html.

28. LEP Higgs Working Group, http://lephiggs . web. cern.ch/LEPHIGGS/ papers/mssm_2001_march/index.html.

29. LEP2 SUSY Working Group, http://lepsusy.web.cern.ch/lepsusy/www/ squarks_moriond01/squarks_pub.html.

30. CLEO Collaboration, M.S. Alam et al., Phys.Rev.Lett. 74 (1995) 2885; S. Ahmed et al., CLEO CONF 99-10; ALEPH Collaboration, R. Barate et al., Phys. Lett. B429 (1998) 169; BELLE Collaboration, K. Abe et al., hep-ex/0103042.

31. G. Degrassi, P. Gambino and G. F. Giudice, hep-ph/0009337; M. Carena, D. Garcia, U. Nierste and C. E. Wagner, hep$\mathrm{ph} / 0010003$.

32. J. A. Casas, A. Lleyda and C. Munoz, Nucl. Phys. B471 (1996) 3; H. Baer, M. Brhlik and D. Castano, Phys. Rev. D54 6944 1996; S. Abel and T. Falk, Phys. Lett. B444 (1998) 427.

33. J. Ellis, T. Falk and K. A. Olive, Phys. Lett. B444, 3; J. Ellis, T. Falk, K. A. Olive and M. Srednicki, Astropart. Phys. 13 (2000) 181.

34. For another treatment of $\chi-\tilde{\tau}$ coannihilation, see: M. E. Gómez, G. Lazarides and C. Pallis, Phys. Rev. D61, 123512 (2000) and Phys. Lett. B487, 313 (2000). For $\chi-\chi^{\prime}-\chi^{ \pm}$ coannihilation, see: S. Mizuta and M. Yamaguchi, Phys. Lett. B298 (1993) 120; J. Edsjo and P. Gondolo, Phys. Rev. D56 (1997) 1879. For $\chi-\tilde{t}$ coannihilation, see: C. Boehm, A. Djouadi and M. Drees, Phys. Rev. D62 (2000) 035012. Neither of the latter processes is important in the CMSSM, though they may be relevant in the more general MSSM.

35. ATLAS Collaboration, Detector and Physics Performance Technical Design Report, http://atlasinfo.cern.ch/Atlas/GROUPS/ PHYSICS/TDR/access.html;

CMS Collaboration, Technical Proposal, http://cmsinfo.cern.ch/TP/TP.html.

36. See S. Abel et al., Tevatron SUGRA Working Group Collaboration, hep-ph/0003154, and references therein.

37. J. Silk and M. Srednicki, Phys. Rev. Lett. 53 (1984) 624 .

38. L. Bergstrom, J. Edsjo and P. Ullio, astro$\mathrm{ph} / 9902012$.

39. L. Bergstrom, J. Edsjo, P. Gondolo and P. Ullio, Phys. Rev. D59 (1999) 043506.

40. J. Silk, K. Olive and M. Srednicki, Phys. Rev. Lett. 55 (1985) 257. 
41. P. Gondolo, talk at the XIXth International Conference on Neutrino Physics and Astrophysics, Sudbury, Canada, June 2000:

http://nu2000. sno.laurentian.ca/.

42. M. W. Goodman and E. Witten, Phys. Rev. D31 (1985) 3059.

43. R. Bernabei et al., DAMA Collaboration, Phys. Lett. B480 (2000) 23.

44. J. Ellis, A. Ferstl and K. A. Olive, Phys. Lett. B481 (2000) 304.

45. R. Abusaidi et al., CDMS Collaboration, Nucl. Instrum. Meth. A444 (2000) 345.

46. J. Ellis, A. Ferstl and K. A. Olive, Phys. Rev. D63 (2001) 065016.

47. For other calculations and more references, see: R. Arnowitt, B. Dutta and Y. Santoso, hep-ph/0101020; H. Baer and M. Brhlik, Phys. Rev. D 57 (1998) 567; A. Bottino, F. Donato, N. Fornengo, S. Scopel, hepph/0010203; A. Corsetti and P. Nath, hepph/0011313; J. L. Feng, K. T. Matchev and F. Wilczek, Phys. Rev. D 63 (2001) 045024.

48. J. Ellis, A. Ferstl and K. A. Olive, in preparation.

49. ALEPH collaboration, R. Barate et al., Phys. Lett. B495 (2000) 1;

L3 collaboration, M. Acciarri et al., Phys. Lett. B495 (2000) 18;

DELPHI collaboration, P. Abreu et al., Phys. Lett. B 499 (2001) 23;

OPAL collaboration, G. Abbiendi et al., Phys. Lett. B499 38.

50. P. Igo-Kemenes, LEP Higgs working group, http://lephiggs . web.cern.ch/LEPHIGGS/ talks/index.html.

51. E. Farhi and L. Susskind, Phys. Rept. 74 (1981) 277.

52. S. Dimopoulos and J. Ellis, Nucl. Phys. B182 (1982) 505.

53. J. Ellis and D. Ross, hep-ph/0012067. 